\title{
Hybridization by Grafting: A New Perspective?
}

Xiumei Zhou

School of Horticulture Landscape Architecture, Henan Institute of Science
and Technology, Xinxiang 453003, China

Yongsheng Liu ${ }^{1}$

School of Horticulture Landscape Architecture, Henan Institute of Science and Technology, Xinxiang 453003, China; and Henan Collaborative Innovation Center of Modern Biological Breeding, Henan Institute of Science and Technology, Xinxiang 453003, China

Additional index words. graft hybridization, horizontal gene transfer, genetics and breeding

Abstract. A recent report demonstrates that entire nuclear genomes can be transferred between plant cells and across the graft junction, resulting in the formation of a new allopolyploid species. This intriguing finding throws a new light on an old problemhybridization by grafting - the subject of heated scientific controversy over the past century.

\section{Hybridization by Grafting: A Long- Standing Debate}

In a recent issue of Nature, Fuentes et al. (2014) reported that entire nuclear genomes could be transferred across the graft junction to generate a species of allopolyploid plant without sexual reproduction. We highly appreciate this intriguing finding. However, it should be noted that their paper contains no reference to Darwin, Winkler, and other previous researchers who have claimed that new species and varieties could arise through grafting. Thus the impression which one gets from reading their paper is that they are inspired by natural grafting and are the first to generate a species by artificial grafting.

The grafting of plants is a very ancient horticultural technique. Although there were many cases of graft-induced variations in ancient China, Charles Darwin was the first to propose the concept of graft hybridization, a type of asexual hybridization in which heritable characters may be induced by grafting (Liu, 2006). Darwin (1868) recorded various alleged cases of the so-called "graft hybrids," in which shoots generated from grafted plants exhibited the characters of both scion and stock. He not only accepted graft hybridization as an established fact, but even speculated on a molecular mechanism by which it might be accomplished. He proposed that cells could "throw off" hereditary particles, which were capable of diffusion through the tissues or from cell to cell, and transmission from parent to offspring. In grafted plants, such diffusion could occur between the cells of the scion and stock. Unfortunately, Darwin's hypothesis of Pan-

Received for publication 25 Nov. 2014. Accepted for publication 26 Feb. 2015.

${ }^{1}$ To whom reprint requests should be addressed; e-mail ysliu63@yahoo.ca. genesis was neither understood nor accepted, and is habitually overlooked.

Trofim Lysenko was a controversial figure in plant science. Although his work on vernalization has evolved into a general framework for plant physiology, his research on genetics was severely criticized by many geneticists. He claimed that environmentally induced changes could be transmitted to the progeny, and that the graft hybrids exhibited a combination of characters from the two species through future generations in the same way that sexual hybrids did (Lysenko, 1943). His claims are now largely thought to be wrong (Hagemann, 2002).

\section{Can Hybrids be Obtained by Grafting?}

An experimental approach to testing the existence of graft hybrids was begun in the early 20th century by Hans Winkler (1907), who made saddle grafts between tomato (Solanum lycopersicum) and nightshade (Solanum nigrum). After union, the scion was removed by a horizontal cut at the junction of stock and scion. Thus adventitious shoots were regenerated from the callus at the graft junction. Most of the shoots were identical to either tomato or nightshade, but a few shoots showed a blending of tomato and nightshade characters (Cowles and Chamberlain, 1911). We know that tomato has $2 n=24$ and nightshade $2 n=72$ chromosomes. Although Winkler never succeeded in producing a graft hybrid having $24+72=96$ chromosomes as he expected, he did obtain several graft chimeras and one graft hybrid he named Solanum darwinianum, which possessed 48 chromosomes. This number was not found in either tomato or nightshade. Winkler himself suggested that fusion had occurred between the nuclei of the two species to give rise to a nucleus containing 96 chromosomes, which was subsequently halved to 48 by some autoregulatory process. But some researchers suggested that it might have been that of a tetraploid form of tomato. At any rate, it was Winkler who coined the term "polyploid" and "genome," and showed that polyploidy plants could be obtained by grafting.

Now Fuentes et al. (2014) have obtained a new species named Nicotiana tabauca, which has a total of 72 chromosomes (24 from N. glauca and 48 from N. tabacum), indicating that allopolyploid species can indeed be generated by grafting, as Winkler expected. It should be noted that the grafting method adopted by Fuentes et al. (2014) is not brand-new. A similar method, which combines plant grafting with tissue culture technique, has been established since 1990s by Japanese researchers (Noguchi et al., 1992). The technical improvements made by Fuentes et al. (2014) are the use of transgenic plants as grafting materials, and variants selection by antibiotics, which seems to be more efficient than Winkler's grafting method.

Intercellular Migration of Nuclei/ Chromatin: A Novel Mechanism for Graft Hybridization?

Although the phenomenon of intercellular movement of nuclei/chromatin was first discovered at the beginning of the 20th century, it was thought to be an artifact. Since 1950s, several Chinese researchers had made a fairly large number of observations on this phenomenon by using various kinds of microscopic techniques and plant materials, demonstrating that it is a naturally occurring and normal event. According to Lou (1963), Linsbauer was the first to consider the intercellular movement of nuclei/chromatin as one mechanism of graft hybridization. Later, Ohta (1991) observed that chromatin masses were indeed transferred from the lignifying and dying cells of the stock to the scion through cell wall and intercellular space. Now Fuentes et al. (2014) have shown that entire nuclear genomes can be transferred between plant cells of the stock and scion. Although we do not doubt its reliability, more experimentation is needed for confirmation. As a reviewer of this paper points out, it is too hasty to make such a conclusion because there is no evidence that the cell wall is intact during the process of grafting and the stage of callus propagation.

In their grafting experimental system, however, Stegemann and Bock (2009) did not observe long-distance movement of genetic materials, and only demonstrated that the genetic exchange was locally confined to the graft junction. This is not sufficient to explain the graft-induced changes we observed in fruit trees. By grafting the scions of purple-leaved plum onto the crown of Yuhuang plum and preventing sexual hybridization between the two species, we observed that there are a number of seedlings of Yuhuang plum (ranging from $2.3 \%$ to $15.8 \%$ ) with the characters of purple-leaved plum (Zhou et al., 2013). It appears to us that there must be long-distance movement of genetic materials in the grafted fruit trees. 
Now the discovery of circulating nucleic acids provides other plausible mechanisms for graft hybridization. Such nucleic acids can not only circulate freely within the whole organism, but can also be integrated into cells, resulting in heritable changes genetically or epigenetically (Gahan, 2013). In the early 1960s, Stroun et al. (1963) succeeded in obtaining the graft hybrids of eggplants, and opened the way for the initiation of the early experiments regarding the circulation of DNA fragments across the graft junction as well as throughout whole plants. It is also well known that mRNA and small RNAs are transported through the grafting system, and travel between plant cells as long-distance information macromolecules (Harada, 2010; Li et al., 2013). This led to the proposal that mRNA molecules derived from the stock cells could possibly be reverse transcribed into cDNA capable of being integrated into genome of the scion cells, resulting in heritable changes in the scion (Liu, 2006). A question is now pressing: Is the mobile RNAs really associated with the heritable changes induced by grafting?

\section{Graft Hybridization as a Means of Plant Breeding}

Commenting on the finding by Fuentes et al. (2014), Jones (2014) stated that "future work built on this research might shed light on whether this process could also lead to the generations of new crops." Actually, during the 1930s and 1960s, extensive experiments on graft hybridization were carried out: Numbers of new crops and varieties were developed by grafting, and hundreds of papers were published in the Soviet Union, China, Japan, and other countries (Liu, 2006). These researches were based on the work of Ivan Michurin, a famous Russian plant breeder, who invented "mentor-grafting" (young seedling may alter its characters in the direction of mature plant on which it is grafted) and "preliminary vegetative approximation" (grafting of parent plants before sexual hybridization) method. Michurin's claim for the methods, which are totally different from Winkler's grafting system, has been independently confirmed by several groups of investigators outside Russia (Liu, 2006; Ohta, 1991; Stroun et al., 1963; Taller et al., 1998).

Michurin made a great many observations and studies of plant life, and discovered hosts of interesting phenomenon and new facts (Michurin, 1949). However, the methods he invented, particularly his "mentor-grafting" method, have not been considered seriously by geneticists and plant breeders. We reaffirm that graft hybridization is a simple and efficient means of plant breeding, and would be especially significant in the improvement of fruit trees for which the life cycle is particularly long and consequently the conventional breeding methods are extremely slow. Moreover, graft hybridization provides striking evidence in favor of Darwin's developmental theory of heredity (Pangenesis), and is critical in revealing the non-Mendelian inheritance pattern in grafted fruit trees. Its reality, if and when fully substantiated, would have a tremendous impact on genetical theory and plant breeding practice. Now Fuentes et al. (2014) have provided us with a novel tool to produce new plant species based on Winkler's grafting system. Further studies are needed to use Michurin's grafting methods and investigate their application potentials and molecular mechanisms.

\section{Literature Cited}

Cowles, H.C. and C.J. Chamberlain. 1911. Graft hybrids and chimeras. Bot. Gaz. 51:147-153.

Darwin, C. 1868. The variation of animals and plants under domestication. John Murray, London, UK.

Fuentes, I., S. Stegemann, H. Golczyk, D. Karcher, and R. Bock. 2014. Horizontal genome transfer as an asexual path to the formation of new species. Nature 511:232-235.
Gahan, P. 2013. Circulating nucleic acids: Possible inherited effects. Biol. J. Linn. Soc. Lond. 110:931-948.

Hagemann, R. 2002. How did German genetics avoid Lysenkoism. Trends Genet. 18:320-324.

Harada, T. 2010. Grafting and RNA transport via phloem tissue in horticultural plants. Sci. Hort. 125:545-550.

Jones, B. 2014. Joining forces-asexual genome merger creates new allopolyploid species. Nat. Rev. Genet. 15:515.

Li, J.X., Y. Wang, L.L. Zhang, B. Liu, L.W. Cao, Z.Y. Qi, and L.P. Chen. 2013. Heritable variation and small RNAs in the progeny of chimeras of Brassica juncea and Brassica oleracea. J. Expt. Bot. 64:4851-4862.

Liu, Y.S. 2006. Historical and modern genetics of plant graft hybridization. Adv. Genet. 56:101129.

Lou, C.H. 1963. Intercelluler movement of protoplasm in higher plants and its physiological significance. Bull. Biol. 5:1-4.

Lysenko, T.D. 1943. Heredity and its variability. Foreign Languages Publishing House, Moscow.

Michurin, I.V. 1949. Selected works. Foreign Languages Publishing House, Moscow.

Noguchi, T., Y. Hirata, and N. Yagishita. 1992. Intervarietal and interspecific chimera formation by in vitro graft-culture method in Brassica. Theor. Appl. Genet. 83:727-732.

Ohta, Y. 1991. Graft-transformation, the mechanism for graft-induced genetic changes in higher plants. Euphytica 55:91-99.

Stegemann, S. and R. Bock. 2009. Exchange of genetic material between cells in plant tissue grafts. Science 324:649-651.

Stroun, M., C.C. Mathon, and J. Stroun. 1963. Modifications transmitted to the offspring, provoked by heterograft in Solanum Melongena. Arch. Sci. 16:225-245.

Taller, J., Y. Hirata, N. Yagishita, M. Kita, and S. Ogata. 1998. Graft-induced changes and the inheritance of several characteristics in pepper (Capsicum annuum L.). Theor. Appl. Genet. 97:705-713.

Winkler, H. 1907. Ueber pfropfbastarde und pflanzliche chimaeren. Ber. Deut. Bot. Ges. 25:568576.

Zhou, X.M., Y.S. Liu, and X.J. Li. 2013. Confirmation of a purple-leaved plum graft hybrid. Genet. Mol. Res. 12:710-713. 